\title{
The protective role of Sestrin 2 in high fat diet-induced nephropathy
}

\section{Abdelali Agouni ${ }^{1}$, Duck Y. Lee ${ }^{2}$, Assaad A. Eid ${ }^{3}$, Yves Gorin², Kumar Sharma ${ }^{2}$}

${ }^{1}$ Department of Pharmaceutical Sciences, College of Pharmacy, QU health, Qatar University, P.O. Box 2713, Doha, Qatar; ${ }^{2}$ School of Medicine, University of Texas Health Sciences Center at San Antonio, San Antonio, TX, USA; ${ }^{3}$ Faculty of Medicine, American University of Beirut, Beirut, Lebanon

\begin{abstract}
Background
$>$ Due to the high prevalence of obesity, the number of cases of diabetes are rising Qatar.

$>$ Diabetes is a major public health problem that affects about $17 \%$ of the Qatari population.

$>$ Diabetes is associated with several metabolic risk factors that contribute to a high rate of micro- and macrovascular events.

$>$ Diabetic nephropathy (DN), is a major complication of diabetes and the leading cause of end stage renal disease and cardiovascular morbidity and mortality.

$>$ Multiple redox-sensitive pathways orchestrate the key pathological events of DN.

> Sestrin 2 (Sesn2), is a novel stress-inducible protein, that suppresses reactive oxygen species and protects from oxidative stress; however, its role in diabetes and its complications is yet to be fully delineated.
\end{abstract}

\section{Aim of the Study}

$>$ Genetic studies showed that Sesn2 contributes to the maintenance of metabolic homeostasis such as normalization of metabolic derangements during obesity and protects cells and organisms from agerelated physiological abnormalities.

$>$ However, the role of Sesn2 in renal physiopathology and in the pathogenesis of diabetic kidney disease and glomerular cell injury associated with diabetes is currently still lacking.

$>$ Therefore, the aim of this study was to assess the impact of Sesn2 deletion on the onset of nephropathy associated with high fat diet (HFD)induced obesity in mice.

\section{Methods}

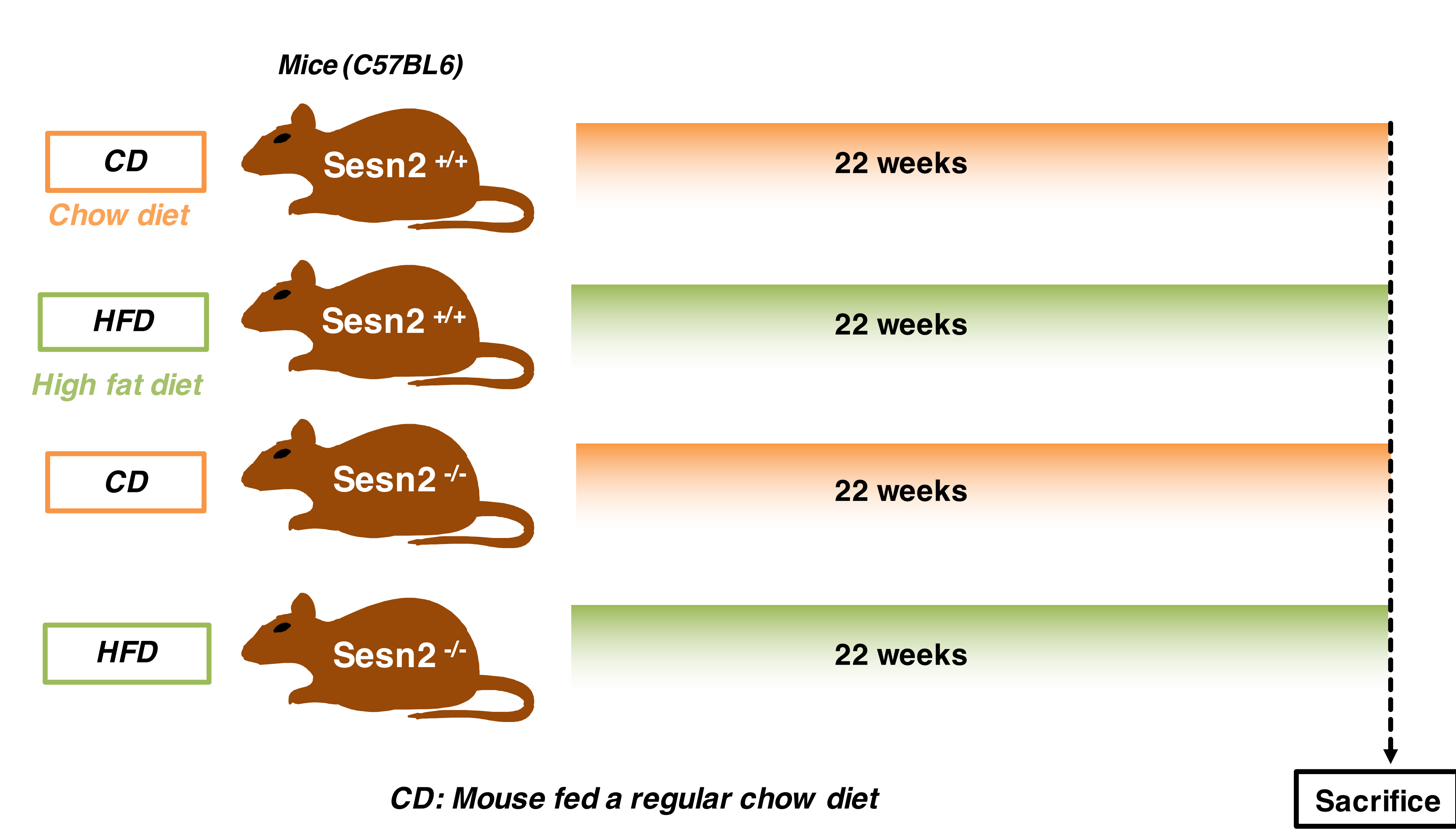
HFD: Mouse fed a high fat diet

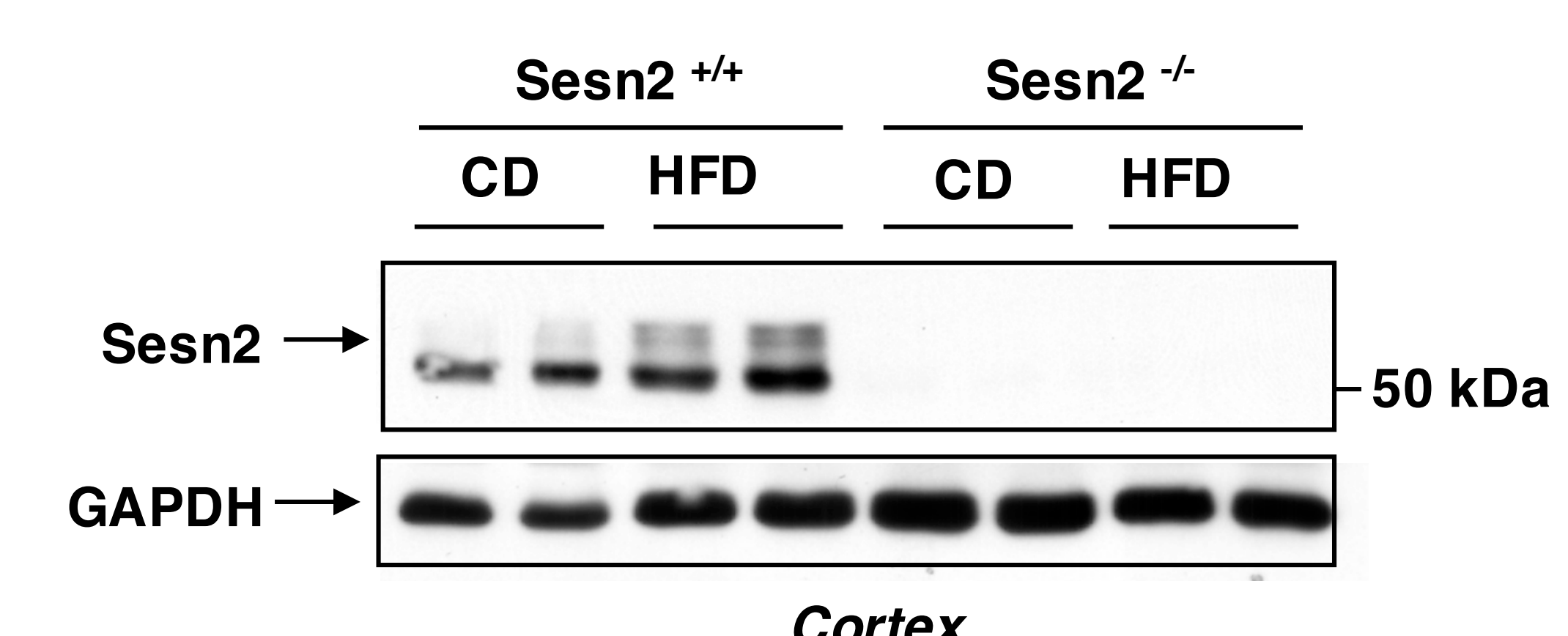

FIGURE 1 | Wild type $\left(\right.$ Sesn2 $2^{+/+}$) and Sesn2-deficient (Sesn2 ${ }^{-/-}$) mice were fed either a chow (CD) or high fat diet (HFD) for 22 weeks, then the structure and function of kidneys from mice were assessed.

\section{Conclusions}

HFD-induced obesity caused upregulation of CD36, an indicator of lipid uptake, and promoted lipogenic enzymes ACLY and FASN, an indicator of de novo lipid synthesis, as well as lipid accumulation in kidney.

$>$ Sesn2 deletion exacerbated HFD-induced renal fibrotic injury

$>$ Taken together, this study provides, for the first time, evidence that Sesn 2 is renoprotective in obesity by diminishing lipid accumulation and blocking excessive lipid uptake and de novo lipid synthesis.

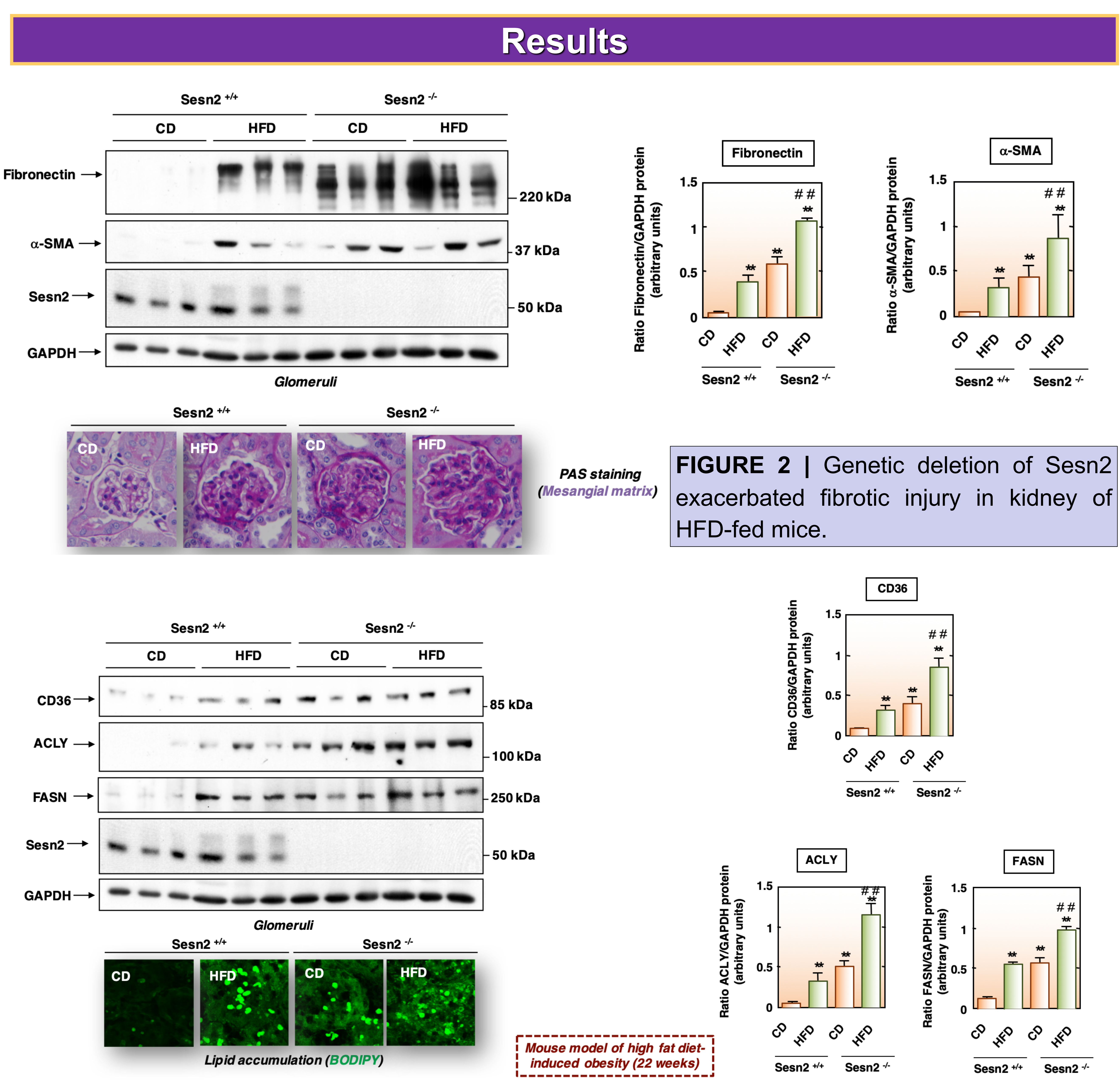

FIGURE 3 | Genetic deletion of Sesn2 enhanced fatty acid translocase (CD36), ATP citrate lyase (ACLY), and Fatty Acid Synthase (FASN) expression and lipid accumulation in kidneys from HFDfed mice.
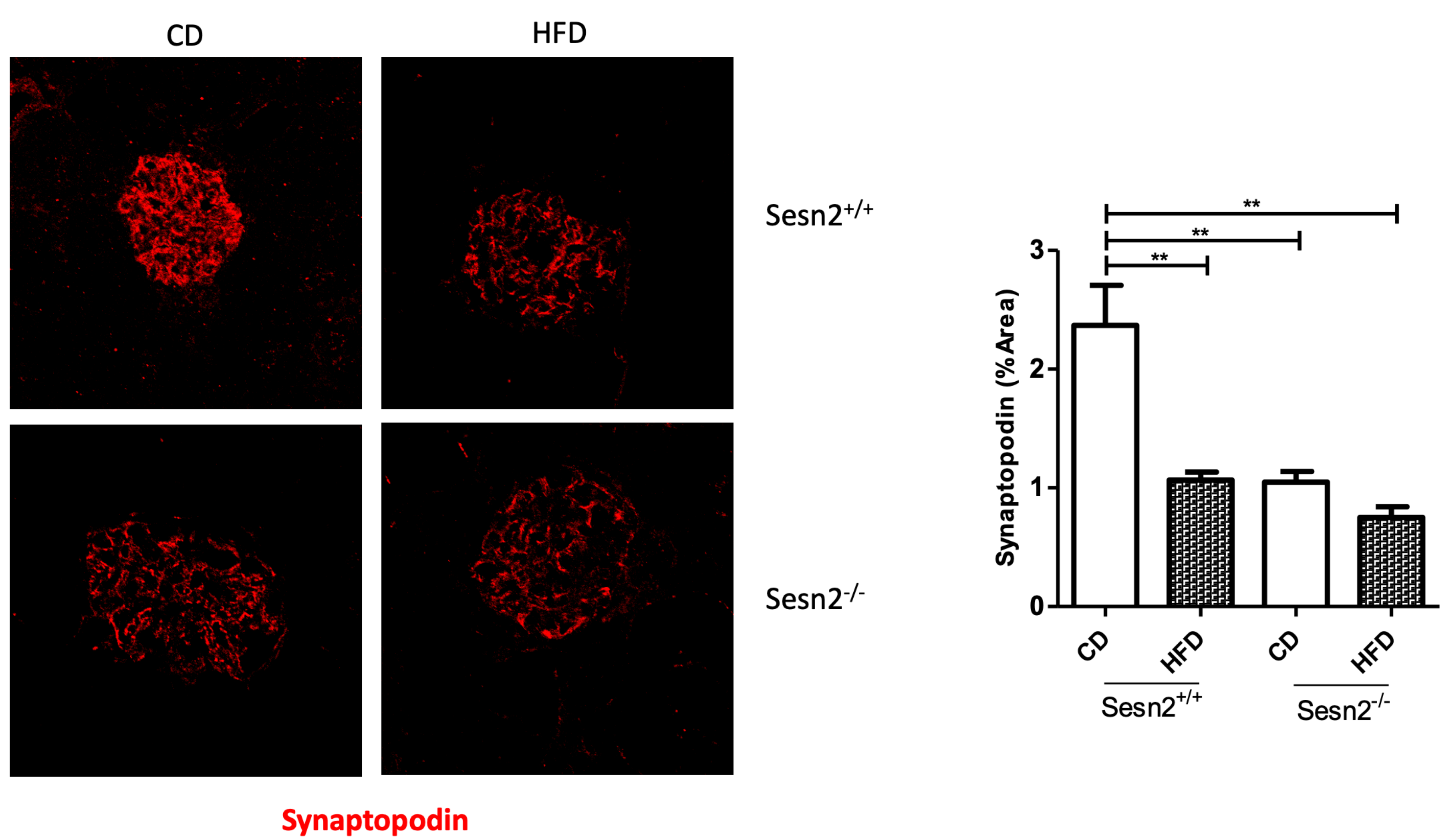

FIGURE 4 | Genetic deletion of Sesn2 damaged podocytes in HFD-fed mice.
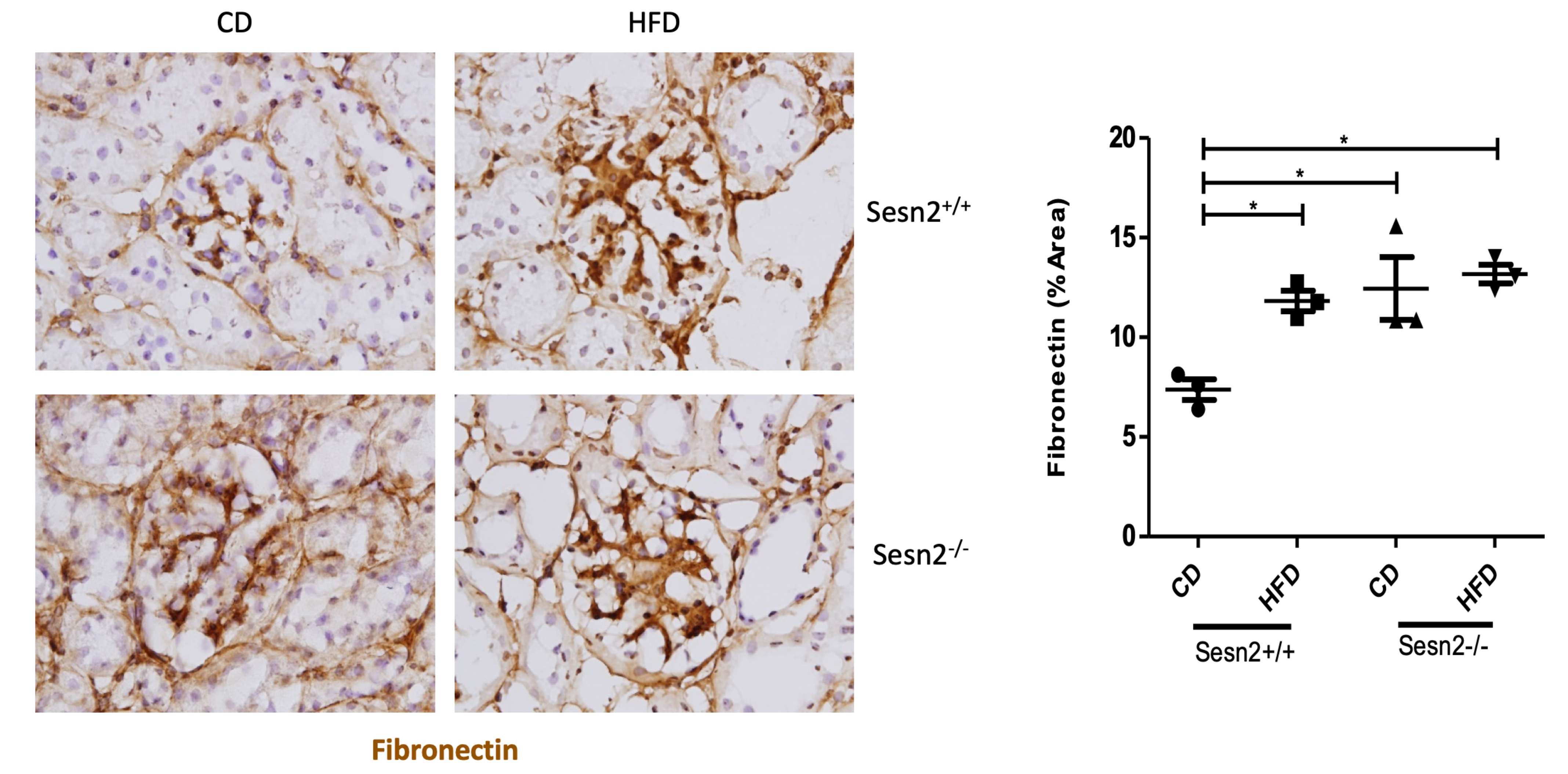

FIGURE 5 | Genetic deletion of Sesn2 aggravated renal fibrotic injury in HFD-fed mice.

\section{Acknowledgements}

This work was supported by award NPRP 8-1750-3-360 from Qatar National Research fund to Dr. Abdelali Agouni. 